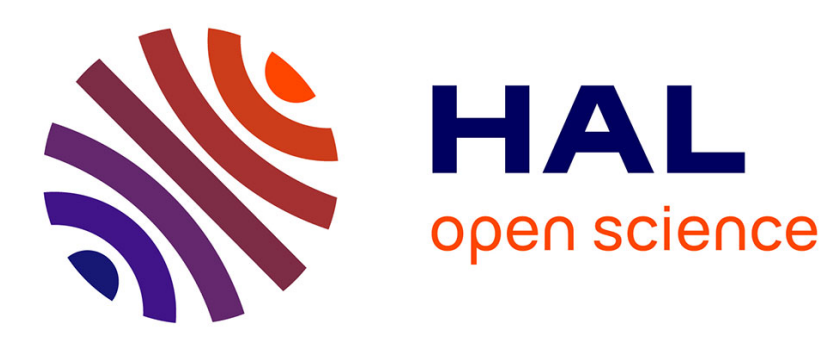

\title{
Low Cost Intelligent System for the 2D Biomechanical Analysis of Road Cyclists
}

\author{
Camilo Salguero, Sandra P. Mosquera, Andres Felipe Barco Santa, Élise
} Vareilles

\section{- To cite this version:}

Camilo Salguero, Sandra P. Mosquera, Andres Felipe Barco Santa, Élise Vareilles. Low Cost Intelligent System for the 2D Biomechanical Analysis of Road Cyclists. ISMIS 2018 - 24th International Symposium on Methodologies for Intelligent Systems, Oct 2018, Limassol, Cyprus. p.130-138, 10.1007/978-3-030-01851-1_13. hal-01923815

\section{HAL Id: hal-01923815 https://imt-mines-albi.hal.science/hal-01923815}

Submitted on 3 Dec 2018

HAL is a multi-disciplinary open access archive for the deposit and dissemination of scientific research documents, whether they are published or not. The documents may come from teaching and research institutions in France or abroad, or from public or private research centers.
L'archive ouverte pluridisciplinaire HAL, est destinée au dépôt et à la diffusion de documents scientifiques de niveau recherche, publiés ou non, émanant des établissements d'enseignement et de recherche français ou étrangers, des laboratoires publics ou privés. 


\title{
Low Cost Intelligent System for the 2D Biomechanical Analysis of Road Cyclists
}

\author{
Camilo Salguero ${ }^{1}$, Sandra P. Mosquera ${ }^{1}$, \\ Andrés F. Barco ${ }^{1[0000-0001-6751-3458]}$, and Élise Vareilles ${ }^{2}[0000-0001-6269-8609]$ \\ 1 LIDIS Research Group, Universidad de San Buenaventura Cali \\ Santiago de Cali, Colombia \\ 2 Industrial Engineering Laboratory, Université de Toulouse, Mines Albi \\ Albi, France \\ Corresponding author: anfelbar@usbcali.edu.co
}

\begin{abstract}
This paper introduces an intelligent system focused on the biomechanical analysis of road bicycle cyclists. This type of analysis is carried out in specialized medical centers that operate using costly resources and are employed, mainly, for studies on athletes of high performance. The proposed system contrasts with these centers in that it provides the rookie cyclist with an accessible and affordable biomechanical analysis, although not as accurate. The architecture of the system rests in the advances in motion capture and augmented reality libraries. In the paper are discussed the motivations of the research, the internal design of the proposed system and the differences with various systems.
\end{abstract}

Keywords: Augmented Reality · Biomechanical analysis · Intelligent Systems · Motion Capture $\cdot$ Road Bicycle $\cdot$ Software architecture

\section{Introduction}

Biomechanics is defined as the study of the movement of living beings using the mechanics analysis, which involves forces as creators of movement [9]. The discipline studies the capture, measurement and analysis of human body movement with the aim of avoiding injuries and improve sports performance. Our research is focused on the movement performed by cyclists in the bicycle route category $[15,12]$, one of the most important sports world-wide.

Frequently beginner cyclists, particularly those of bicycle route category, have a bad or uncomfortable posture when using their bicycles which generally has a negative impact on their performance [15]. This situation is due to unknowing of the correct positioning on the bicycle that may lead to an optimal performance. Normally, beginner cyclists come to good postures only through practice and imitation of positioning of professional or high performance athletes. This imitation of expert cyclists is not straightforward as differences on positioning cannot be observed with the naked eye and much less if the cyclist is riding.

Biomechanical studies with traditional systems carried out in specialized centers are usually done in controlled environments, mainly with controlled illumi- 
nation, with several cameras and specialized sensors and with non versatile proprietary software [8]. In addition to this, access to a biomechanical evaluation is impossible for many cyclists in developing countries as it is an expensive service. In Colombia, for instance, where road cycling is one of the national sports, it exists only two biomechanical centers (visit $[14,4]$ for details in Spanish) that performs an evaluation that cost around 250 US Dollars (around 750.000 COP), which is not affordable for most beginners cyclists.

Our goal is to develop a versatile and portable biomechanical analysis intelligent systems that contrasts with specialized center systems in that it does not require expensive devices or complex features. Given the low cost characteristic, the system does not have the same precision in its bicycle and cyclist measurements and is not able to implement all the biomechanical analysis techniques that standard systems can perform. However, it does strengthen the versatility with which athletes can improve their positioning on their bicycles

The rest of the document presents the following topics. Related work of systems that currently use low resources are presented in Section 2. The design of an intelligent system for the biomechanical analysis is discussed in Section 3. Afterwards, the advantages and disadvantages of our proposal are shown in Section 4. Finally, conclusions and future work are drawn in Section 5.

\section{Related Work}

In this section we present related work of systems for the biomechanical analysis that use of low resources but that are not fully automatic systems, meaning that the human intervention is required, given opportunity to configuration failures and complication.

The first related work is called Kinematic analysis of cyclist pedaling using systems of motion capture in $2 D$ and $3 D$ [5]. This research focused on checking the reliability of the data thrown by a low cost analysis system that works in $2 \mathrm{D}$ and compares it with a system that works in 3D that makes use of different expensive resources. The study was carried out through the simultaneous use of both systems (2D and 3D) over a group of 12 cyclists, and the subsequent comparison of the data offered by both systems. Among the results and conclusions, authors did not find important differences between the sensitivity of both systems. On the other hand, as a drawback in the 2D measurement systems, the data obtained for the evaluation was not captured in real time, a characteristic of the $3 \mathrm{D}$ systems. The intelligent system that we propose works as well in $2 \mathrm{D}$ but it does operates real time, which addresses the disadvantage of the 2D system.

A second work on the subject is found in [11] and it is called Biomechanical analysis for cyclists. The research focuses on the analysis of the position of the lower joints of the rider. The authors developed an application for Android systems using the Java programming language and the OpenCV library [13]. To make use of their system, the cyclist must locate LED bulbs in his lower joints and record a video of the cyclist performing the pedaling movement on the bicycle. Finally, the system presents graphs with the data of the angles of the 
cyclist's joints movement. In contrast with this system, our system does not force the uses of LED bulbs but whatever color marker that facilitates the motion capture process. Additionally, the capture is done in real time without the need of an intermediate video.

Another work on biomechanical analysis is found under the name Mobile tracking system and optical tracking integration for mobile mixed reality [3]. This work aims at developing a system that combines optical tracking and augmented reality, operating in a local area network as it communicates with a server responsible for processing all tracking data. The idea behind it is to reduce the responsibilities of the mobile device that have limited processing and storage capabilities. The system is possible thanks to the implementation of a fast communication channel between mobile device and servers.

Apart from the described works, there are commercial systems that allow cyclists to perform a biomechanical analysis of low cost. To the best of the authors knowledge, these applications do not have related academic publications in which the underlying models and techniques are described.

Bike Fast Fit [1] is an application available for iOS devices, which allows to capture in video the pedaling of the cyclist to subsequently measure the angles presented in the recording. This measurement is done to a certain degree manually, meaning that the user has to choose the frames of the video where it is potentially correct to take the measurement. Also the user must choose the body angle to measure, and then locate the measurement points in the cyclist's joints. The advantage of this system is that it has a good precision in the measurements. Nonetheless, the precision may be affected by the intervention of the user as it can make mistakes when measuring and configuring the system.

The Roadie Bike Fit [2] is an application available for Android devices, which offers the user a guide and recommendations for the adjustment of the bicycle. The guide begins with the capture of two images, one of them of the bicycle and another of the cyclist. With these two images the application executed an object recognition algorithm with which it identifies body angles and bicycle measurements. The automation and guidance feature make of this a simple and intuitive application for the user. Nevertheless, the measurements are obtained from static positions of the cyclist, which does not give reliable data of the movements that can only be measured while the cyclist pedals.

\section{System's Underlying Design}

\subsection{Solution Scheme}

In the face of the problem, the proposed system is able to offer the route cyclist a kinematic analysis and recommendations to adapt the bicycle and body to their correct positions. This is done through the measurement of the body angles involved in the movements. The measurements are performed mostly in the sagittal plane (refer to [7] for information about different positions to capture movement data). Thus, our system uses a camera perpendicular to the direction of the bicycle, focusing on the cyclist's right profile, as shown in Fig. 1.a. 


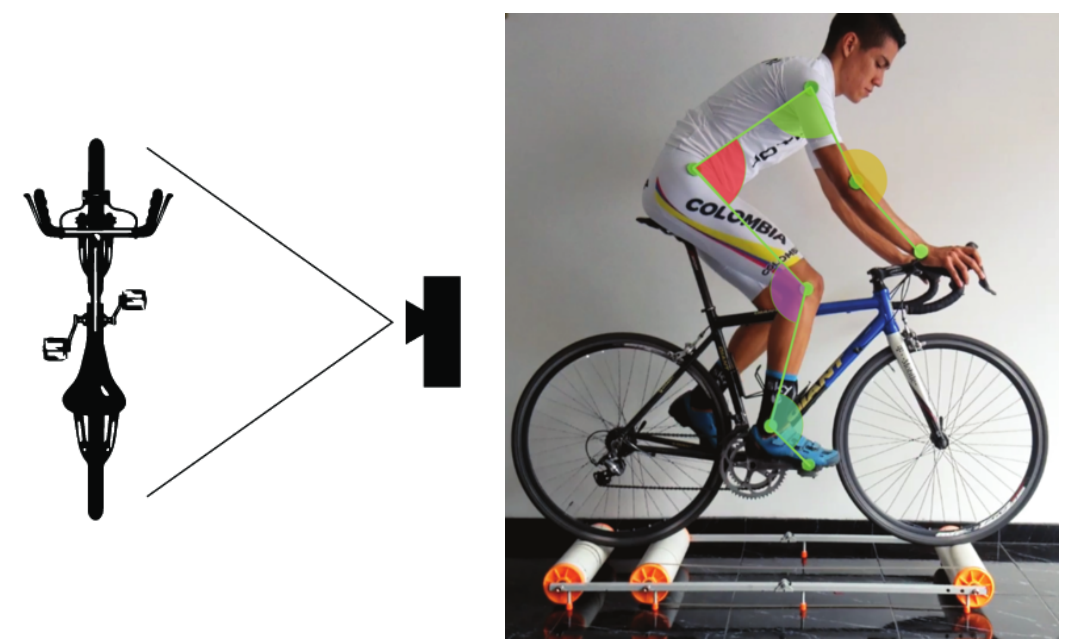

Fig. 1. a) Camera captures right profile (sagittal plane). b) Angles in the sagittal plane involved in the movement.

Having this configuration, the system captures the data necessary to execute the biomechanical analysis, i.e., the angles formed by the body parts. As presented in Fig. 1.b, in order to measure the angles, the cyclist must locate markers of any color that are distinctive in the image (high contrast to improve image recognition). The system interaction is as follows. Once the capturing device (e.g., smart-phone camera) is in front of the cyclist, it starts marker recognition on the video stream. Using the frames of the video stream it computes angles (as we will see further in Section 3.2). Then the angles are rendered over a display and finally a recommendation based on an analysis is given.

\subsection{Architecture}

The intelligent system implements the architecture presented in Fig. 2. Essentially, the application executes the biomechanical analysis on a device (such as a tablet, mobile device or laptop) and statistics are stored on a server.

Client's side. The client's components are responsible for the most important tasks of the system, the core of the system; motion capture, data processing (biomechanical analysis) and visualization of results.

- Capture: This component captures the movement from the right profile of the cyclist. It uses only one video capturing device as a smart-phone camera and no sensors, making it low cost. The video stream is presented in a first HTML5 canvas.

- Processing: The processing of the captured images are performed on the client's side, making use of the library tracking.js [6]. The library inte- 


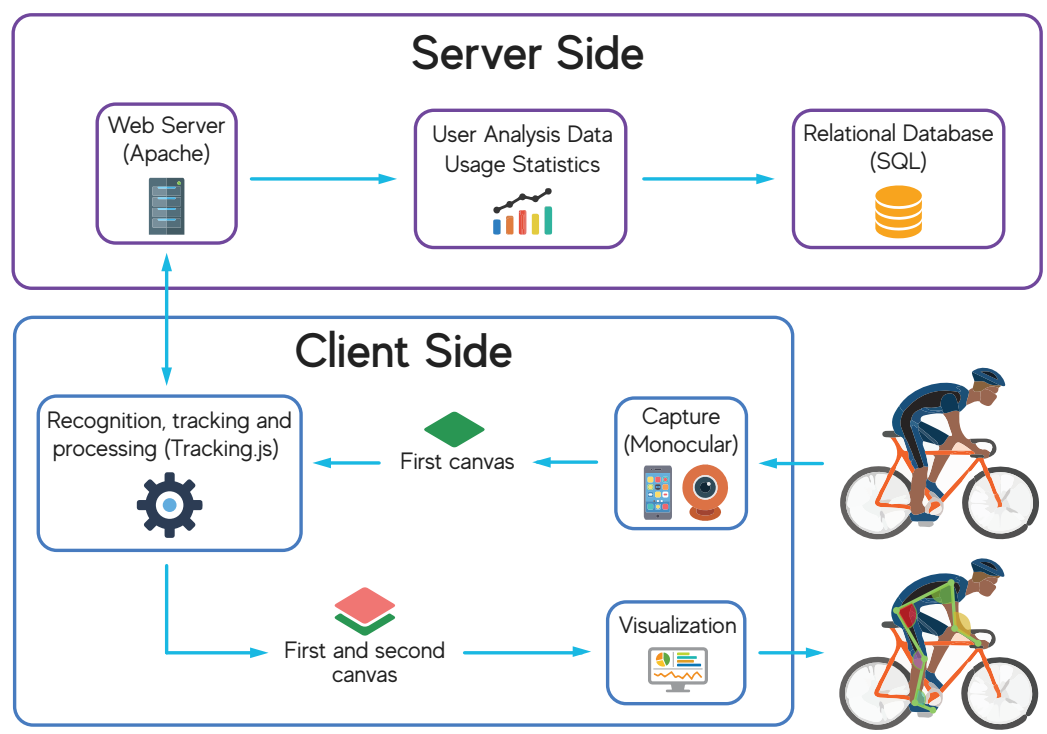

Fig. 2. System's architectural design.

grates functionalities of image recognition and tracking of colors thus allowing to capture in real-time the position of the color markers that the cyclist is wearing. Having captured the positions of the markers, which are points in the vector space $R^{2}$, the system proceeds with the computation of angles. To do so two vectors are need to be computed (as presented in Fig. 3) by using three pixels coordinates (from the markers) and applying basic vector geometry equations.

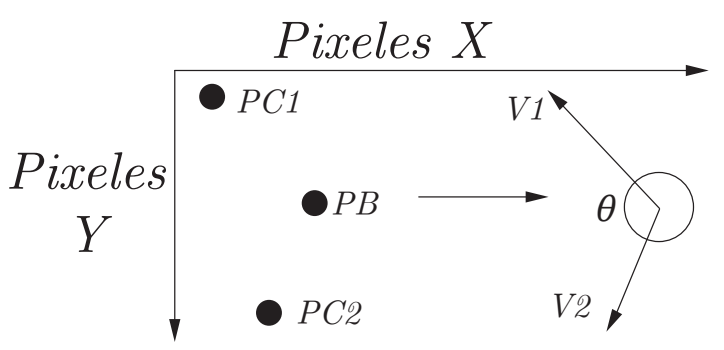

Fig. 3. Obtaining vectors from point in vector space $R^{2}$.

Once the vectors $\overrightarrow{V 1}$ and $\overrightarrow{V 2}$ are computed, the system computes the vectors' magnitudes (Equations 1 and 2), computes the dot product using its 
algebraic notation (Equation 3) and finally it computes the angle using the dot product geometry notation (isolating $\theta$ in Equation 4).

$$
\begin{gathered}
|\overrightarrow{V 1}|=\sqrt{V 1 x^{2}+V 1 y^{2}} \\
|\overrightarrow{V 2}|=\sqrt{V 2 x^{2}+V 2 y^{2}} \\
\overrightarrow{V 1} \cdot \overrightarrow{V 2}=V 1 x \times V 2 x+V 1 y \times V 2 y \\
\overrightarrow{V 1} \cdot \overrightarrow{V 2}=|\overrightarrow{V 1}| \times|\overrightarrow{V 2}| \times \cos \theta
\end{gathered}
$$

Finally, the biomechanical analysis of the cyclist is done by using cyclist and bicycle measures as well as the computed angles. This analysis is under implementation in the system as it requires the knowledge from experts in the field to be extracted.

- Visualization: The visualization is done in a fully augmented reality fashion. This technology integrates information captured from the real world with digital information, matching the two of them to build a new coherent complemented and enriched world [10]. The system does so by rendering information related to the positioning of the body and presenting recommendations of modification tailored to the bicycle. The data resulting from the analysis together with the bicycle adjustment recommendations are rendered in real time in a second HTML5 canvas.

Server's side. In the server' side are implemented the persistent storage for statistics on the systems' use.

- Storage: The server is in charge of storing information about the biomechanical analysis made by cyclists. So far, the system does not manage users as it can be executed without sessions but it is planned to add such functionality. Nevertheless, it stores users data and results for future statistical analysis.

- Web Server: The server processes the collected data from users and analysis results in order to obtain use statistics. These statistics refer to the most common positioning errors made by novice cyclist, the number of times a given cyclist uses the tool, measurements of bicycles and execution time. This data is planned to be exploited with data mining algorithms in order to improve recommendations.

\section{Key Differences with Other Systems}

There exist some clear differences with respect to the systems in specialized centers and also with commercial systems available for mobile devices. We expose these as advantages and disadvantages.

1. One advantage w.r.t. systems developed as part of research or as a commercial product, is that out system requires very little configuration work on the part of the user. This is because the motion capture of the joints is highly automated, requiring only to select the appropriated color for the markers that the cyclist is wearing. 
2. By having augmented reality as a visualization technology, the information related to the analysis is clearly displayed and easily understandable by the cyclist. This contrasts with the results in specialized centers that must be interpreted by experts.

3. The intelligent system offers the cyclist graphic recommendations, with the use of augmented reality, so that it can autonomously adjust the bicycle and thus implement a proper posture. No mechanics or expert intervention.

4. The system is implemented using a library that works in web environments, providing flexibility and a multi-platform development. Other systems either have too complex environment or are tied with a given platform.

5. The system uses only a video capture device and a processing unit (e.g. a smart-phone). Ergo, it is low cost unlike specialized centers.

Our system, on the other hand, presents the following disadvantages.

1. First, the system is affected in its measurements by the processing speed because it is designed to perform real-time analysis on a mobile devices that have limited processing and storage capabilities. It can therefore be slower than traditional systems.

2. Secondly, the angle and position of the camera may influence the reliability of the captured data which may generate inaccurate measurements.

3. Since it is a system that identifies the joints from markers, these markers are recognized by the system using an specific color. This process, made by the library tracking.js, is error prune and depends mostly on the color composition of the image (cyclist, bicycle and surroundings). Not being able to identify the marker implies not joints movement capture.

4. Finally, adequate lighting can be a determinant factor when capturing data. Unlike the specialized centers where there is a controlled environment, the lighting when using the proposed application may vary vastly in different executions making the analysis difficult or inaccurate.

\section{Concluding Remarks}

In this paper we have presented the motivations and internal design of an intelligent system focused on the biomechanical analysis of road bicycle cyclists. In particular, the paper briefly discussed the context of cycling, the intrinsic need of novice athletes for analysis of positioning and movement and related work found in the literature and Web. Our research is possible given the advances in mobile devices (cameras), their access, high-speed WI-FI networks and advances in libraries for motion capture and augmented reality. As such, the system is an important starting point for the novice cyclists as it allows to; do motion capture (thanks to the library tracking.js); show the cyclist movement over the display in real time (thanks to the augmented reality technology), do the biomechanical analysis in real time using the limited processing and storage capabilities of a mobile device, store usage statistics for further analysis and be used in a friendly and intuitive way. 
Future work. We have identified three strategic directions for future work.

- First, increase the accuracy of the analysis with the use of sensors. This step is fundamental for improving the analysis and is possible thanks to the inclusion of sensors in the latest mobile devices (mainly smart-phones).

- Second, it is important to dwell more on usage statistics. Here, it is planned to make a analysis of the stored data (how many times a user executed the system, measurements, etc.) in the aim of providing good recommendations for the novice cyclist. In short, it is planned to apply data mining.

- Lastly, it is planned to involve a knowledge base that helps the positioning of cyclist either by applying expertise knowledge or case-based reasoning.

\section{References}

1. Bike fast fit, https://goo.gl/AQP9vM, App Store, Last access 26 April 2018

2. The roadie bike fit, https://goo.gl/cNbPxE, Play Store, Last access 26 April 2018.

3. Bajana, J., Francia, D., Liverani, A., Krajčovič, M.: Mobile tracking system and optical tracking integration for mobile mixed reality. Int. J. Comput. Appl. Technol. 53(1), 13-22 (Dec 2016). https://doi.org/10.1504/IJCAT.2016.073606

4. Biomec: Bikefitting 3d, http://www.biomec.com.co/bikefitting.html, Last access 26 Abril 2018.

5. Abal del Blanco, P.: Análisis cinemático del pedaleo ciclista mediante sistemas de captura de movimiento en 2d y 3d (2016), Universidad de León

6. Community, T.: Tracking.js, https://trackingjs.com/, Last access 26 April 2018.

7. Ferrer Roca, B.: Comparación de diferentes métodos de ajuste de la bicicleta en ciclistas entrenados. influencia de factores biomecánicos y energéticos (2015), Universidad de León

8. John, S., Andrew, Z.: The economic impact of sports facilities, teams and mega-events. Australian Economic Review 39(4), 420-427. https://doi.org/10.1111/j.1467-8462.2006.00431.x

9. Knudson, D.: Fundamentals of Biomechanics. SpringerLink: Springer e-Books, Springer US (2007)

10. van Krevelen, D.W.F.R., Poelman, R.: A survey of augmented reality technologies, applications and limitations. International Journal of Virtual Reality 9(2), 1-20 (Jun 2010)

11. Márquez, C.J., Pérez, L., Tocino, D.: Análisis biomecánico para ciclistas (2017), Universidad Complutense de Madrid

12. Mota, L.N., Junior, A.J., Neto, G.A., Mota, E.N.: Influence of bike fit in lower limb biomechanics in road cyclists. Physical Therapy in Sport 31, e7 (2018). https://doi.org/https://doi.org/10.1016/j.ptsp.2017.11.031

13. Pulli, K., Baksheev, A., Kornyakov, K., Eruhimov, V.: Real-time computer vision with opencv. Commun. ACM 55(6), 61-69 (Jun 2012). https://doi.org/10.1145/2184319.2184337

14. SCIENCE, A.S.: http://www.aethosport.com/laboratorio, Last access 26 April 2018.

15. Too, D.: Biomechanics of cycling and factors affecting performance. Sports Medicine 10(5), 286-302 (Nov 1990). https://doi.org/10.2165/00007256199010050-00002 